\title{
Nonequilibrium Atmospheric Plasma Jets Assisted Stabilization of Drug Delivery Carriers: Preparation and Characterization of Biodegradable Polymeric Nano-Micelles with Enhanced Stability
}

\author{
Shogo Sumitani ${ }^{1}$, Hiroki Murotani ${ }^{1}$, Motoi Oishi ${ }^{1-3,5}$, Katsuhisa Kitano ${ }^{6}$, \\ Satoshi Hamaguchi ${ }^{6}$, Yukio Nagasaki*1-5
}

${ }^{1}$ Institute of Materials Science, Graduate School of Pure and Applied Sciences,

${ }^{2}$ Tsukuba Research Center for Interdisciplinary Materials Science (TIMS),

${ }^{3}$ Center for Tsukuba Advanced Research Alliance (TARA),

${ }^{4}$ Master's School of Medical Sciences, Graduate School of Comprehensive Human

Sciences, University of Tsukuba, Tennoudai 1-1-1, Tsukuba, Ibaraki 305-8573, Japan

${ }_{5}^{5}$ Satellite Laboratory, International Center for Materials Nanoarchitectonics (MANA),

National Institute for Materials Science (NIMS) and University of Tsukuba,

Tennoudai 1-1-1, Tsukuba, Ibaraki 305-8573, Japan

${ }^{6}$ Center for Atomic and Molecular Technologies, Graduate School of Engineering, Osaka University, Yamada-oka 2-1, Suita, Osaka 565-0871, Japan

Key words: poly(ethylene glycol)-block-poly(lactide) copolymer, DDS, polymeric micelles, core-polymerization, plasma jets, dielectric barrier discharge plasma,

\section{Introduction}

Polymeric micelles formed in aqueous media through self-assembly of poly(ethylene glycol)-block-poly(lactide) copolymers (PEG- $b$-PLA) have been extensively studied as drug delivery carriers, because PEG- $b$-PLA micelles possessing FDA-approved polymer components showed high biocompatibility and applicable biodegradability ${ }^{1-3}$. Their hydrophilic PEG shells serve as a brush to avoid the protein adsorption and subsequent non-specific uptake by reticuloendothelial systems (RES) after intra-venous injection, and their hydrophobic PLA cores accommodate drugs with a high loading efficiency.

The PEG- $b$-PLA micelles are known to possess a relatively low critical association concentration (CAC) value and exhibit a slow dissociation behavior different from the micelles composed of low molecular weight surfactant even under dilute conditions ${ }^{4}$. However, the physical assemble force, i.e. intermolecular hydrophobic and/or van der Waals interaction; as the main driving force to form the micelle with core-shell structure may not be enough to maintain its stable form under in vivo conditions.
Consequently, the dissociation of the micelles occurs below the CAC in the bloodstream, causing low therapeutic efficacy and side-effect arisen from non-targeted release of the drugs.

To this end, many studies have been focused on the stabilization of micellar structures by the polymerization or chemically cross-linking of either shell or core segmnets ${ }^{5,6}$. However, these approaches are required to entrap cross-linkers or thermo- and photo-radical initiators into micelle using organic solvent, resulting in a decrease in the drug loading capacity. Recently, we have reported an alternative approach to generate reactive species (hydroxyl radicals and superoxide) in aqueous media by nonequilibrium atmospheric plasma jets. For example, irradiation of plasma jet to a solution of $\mathrm{HAuCl}_{4}$ afforded the gold nanopraticles without any additional reducing agents through the reduction of $\mathrm{Au}(\mathrm{III})$ ions to $\mathrm{Au}(0)$ caused by the reactive species ${ }^{7}$. For other instance, methacrylic monomers were polymerized in the absence of radical initiator in aqueous media at room temperature under irradiation of nonequilibrium atmospheric plasma jets $^{8}$, strongly suggesting that methacryloyl group serves as plasma-sensitive functional group even 
under the mild plasma jet conditions. In this study, we describe a novel strategy for the preparation of core-polymerized PEG- $b$-PLA micelles using nonequilibrium atmospheric plasma jets. Thus, PEG- $b$-PLA micelles self-assembled from PEG- $b$-PLA bearing an acetal group at PEG end and a methacryloyl group at PLA end (acetal-PEG- $b$-PLA-MA) were polymerized by irradiation of plasma jets (Figure 1). Eventually, the core-polymerized PEG- $b$-PLA micelles show the enhanced stability against organic solvent and lower CAC value compared with that of conventional (non-polymerized) PEG- $b$-PLA micelles. We believe that use of nonequilibrium atmospheric plasma jets may represent a promising approach for the preparation of stable polymeric micelles, because this process dose not require neither cross-linkers nor thermo- and photo-radical initiators.

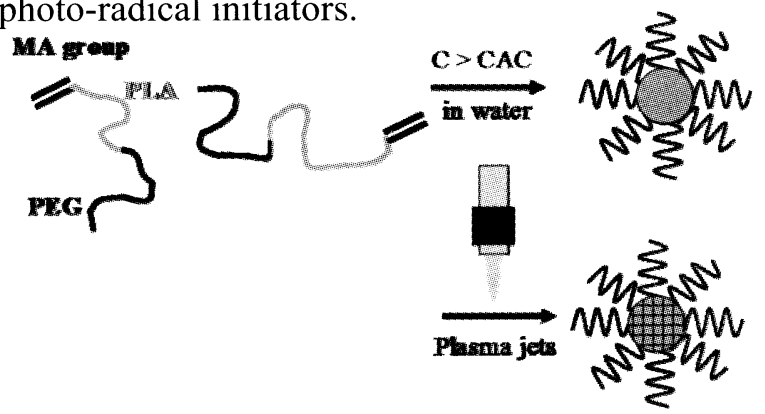

Figure 1. Schematic illustration of preparation of core-polymerized PEG- $b$-PLA-MA micelles using plasma irradiation.

\section{Experiments}

\subsection{Materials and methods}

Tetrahydrofuran (THF) was purified by conventional distillation under nitrogen atmosphere. Azobisisobutironitrile (AIBN) was recrystallized from methanol. $N, N$-Dimethylacetamide (DMAc) and pyrene were used as received. Gel permeation chromatography (GPC) measurements were carried out using $\mathrm{TOSOH}$ HLC-8120 equipped with TSK gel columns (Super HZ4000 and Super HZ3000) and an internal refractive index (RI) detector using THF with $0.5 \%(\mathrm{v} / \mathrm{v})$ of triethylamine as an eluent at a flow rate of $0.35 \mathrm{~mL} \mathrm{~min}{ }^{-1}$ at $40{ }^{\circ} \mathrm{C}$. ${ }^{1} \mathrm{H}$-NMR spectra were obtained in chloroform- $d$ with JEOL EX270 spectrometer at $270 \mathrm{MHz}$. Acetal-PEG- $b$-PLA-MA (Scheme 1) was synthesized as described previously ${ }^{9}$. In this study, the molecular weight of the PEG segment and the polymerization degree of the PLA segment of the block copolymer were $9,500 \mathrm{~g} / \mathrm{mol}$ and 69 , respectively. The quantitative (> $95 \%$ ) functionality of both acetal and methacryloyl end groups was confirmed by ${ }^{1} \mathrm{H}$ NMR data. Acetal-PEG- $b$-PLA was also synthesized as described previously ${ }^{4}$ (PEG; $M_{\mathrm{n}}=6,600$, PLA units; 68).

\subsection{Preparation of polymeric micelles}

Acetal-PEG- $b$-PLA-MA or acetal-PEG- $b$ PLA was dissolved in DMAc at the concentration of $7.0 \mathrm{mg} / \mathrm{mL}$. The solution $(20 \mathrm{~mL})$ was dialyzed against distilled water $(2.0 \mathrm{~L})$ for $24 \mathrm{~h}$ using a pre-swollen semi-permeable membrane (Spectra/Por 2, molecular weight cut-off 3,500 SPECTRUM, Houston, USA) to form the micelle. The water was exchanged at 2, 4 and $8 \mathrm{~h}$ from the beginning. For comparison, core-polymerized PEG- $b$-PLA-MA micelle was prepared by free radical polymerization using AIBN, as previously reported $^{9,10}$.<smiles>C=C(C)C(=O)OC(C)C(=O)OCCOCCOCCC(O)O</smiles>

Scheme 1. poly(ethylene glycol)-block-poly(lactide) with acetal group at PEG chain end and methacryloyl group at PLA chain end (acetal-PEG- $b$-PLA-MA)

\subsection{Irradiation of plasma jets to polymeric} micelles

A schematic diagram of the plasma jets apparatus is shown in Figure 2. Plasma used in this study was generated by $6.5 \mathrm{kV}$ with $10 \mathrm{kHz}$ pulsed high voltage applied to the electrode with He gas flow $9 \mathrm{~L} / \mathrm{min}$. Plasma was irradiated to 5 $\mathrm{mL}$ of micelle solutions $(2.0 \mathrm{mg} / \mathrm{mL})$ for $5-60$ minutes at room temperature.

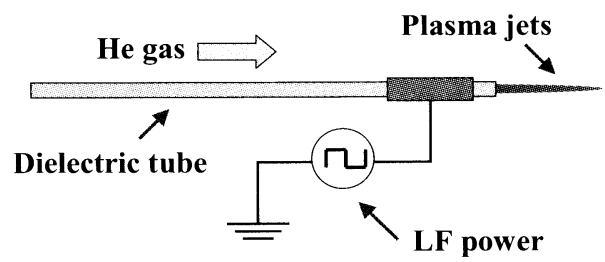

Figure 2. Illustration of low frequency dielectric barrier discharged plasma jet apparatus.

\subsection{Characterization of micelles}

The average diameter of the micelles was determined by dynamic light scattering (DLS) measurements at $25{ }^{\circ} \mathrm{C}$ using $\mathrm{He}-\mathrm{Ne}$ laser at 633 nm (Zetasizer NANOZS, Malvern, UK). The stability of the micelles was evaluated by light scattering intensity of micelles in a mixture of water and THF $(33 \% \mathrm{v} / \mathrm{v})$ solutions. After irradiation of plasma jets to 
acetal-PEG- $b$-PLA-MA or acetal-PEG- $b$-PLA micelle solutions $(0.5 \mathrm{~mL}$ of $2.0 \mathrm{mg} / \mathrm{mL})$ for $5-40$ min, $1 \mathrm{~mL}$ of THF was added to a micelle solution to dissociate the micelle. After that, measurement of light scattering intensity of the solution at $25{ }^{\circ} \mathrm{C}$ was carried out using $\mathrm{He}-\mathrm{Ne}$ laser at $633 \mathrm{~nm}$ (Zetasizer NANOZS). According to the literature ${ }^{10}$, the critical association concentration (CAC) of the micelles was determined using pyrene as a fluorescent probe to monitor the change in the polarity of the core of the micelles.

\section{Results and discussion}

\subsection{Preparation of micelles by dialysis method}

The polymeric micelles were prepared from acetal-PEG- $b$-PLA-MA or acetal-PEG- $b$-PLA by means of dialysis method, as described in the Experimental section. Figure 3 shows the size distribution of the obtained acetal-PEG- $b$-PLA-MA micelle. The average diameter and polydispersity index (PDI) of acetal-PEG- $b$-PLA-MA micelle determined by DLS measurement were $43.9 \mathrm{~nm}$ and 0.144, respectively. The average diameter and PDI of acetal-PEG- $b$-PLA micelle were $37.6 \mathrm{~nm}$ and 0.112 , respectively (data not shown), indicating that both micelles have similar average diameter and PDI.

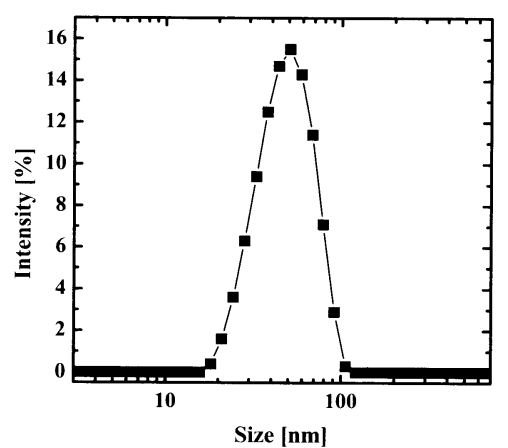

Figure 3. Size distribution of acetal-PEG- $b$-PLA-MA micelles.

\subsection{Reactivity of acetal-PEG-b-PLA-MA for plasma jets}

To clarify the reactivity of the methacryloyl group in the core of the acetal-PEG- $b$-PLA-MA micelle, irradiation of plasma jet was carried out for 5, 20, and $40 \mathrm{~min}$. The resulting acetal-PEG- $b$-PLA-MA micelle solutions were lyophilized, and then ${ }^{1} \mathrm{H}-\mathrm{NMR}$ measurements were carried out in $\mathrm{CDCl}_{3}$ as a good solvent for PEG- $b$-PLA-MA to observe the peak of methacryloyl group. Figure 4 shows the conversion of methacryloyl group in the core of the acetal-PEG- $b$-PLA-MA micelle after irradiation of nonequilibrium atmospheric plasma jets. Note that the conversion of methacryloyl group increased dependent on plasma irradiation time, viz., about $18 \%$ conversion of methacryloyl groups was observed after irradiation for $40 \mathrm{~min}$. This fact indicates that the methacryloyl group in the core of the acetal-PEG- $b$-PLA-MA micelle also has susceptibility for the reactive species generated by plasma irradiation.

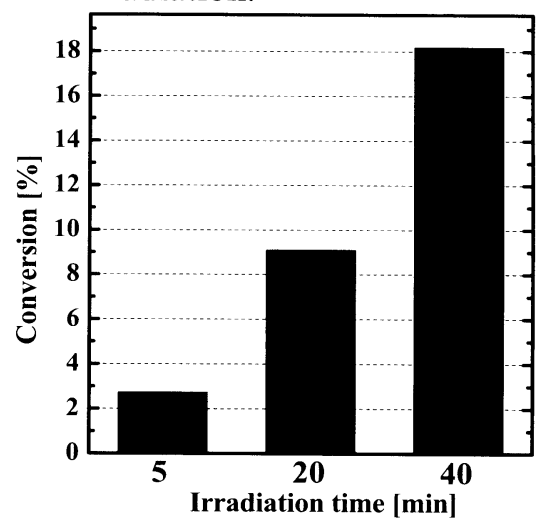

Figure 4. Conversion of methacryloyl group of acetal-PEG- $b$-PLA-MA versus plasma irradiation time.

Figure
shows GPC
chromatograms
of the
lyophilized
acetal-PEG- $b$-PL
$\begin{aligned} & \text { A-MA samples } \\ & \text { before and after } \\ & \text { plasma }\end{aligned}$ irradiation for 40
irradiation, the peak of unimer $\left(M_{\mathrm{n}}=15,700\right.$, $M_{\mathrm{w}} / M_{\mathrm{n}}=1.12$ ) was only observed at ca. $10 \mathrm{~min}$. On the other hand, the plasma-irradiated acetal-PEG- $b$-PLA-MA showed the peaks at higher-molecular-weight position (ca. $5.5 \mathrm{~min}$ : $M_{\mathrm{n}}>3,000,000$ and 7.8-8.5 min: $M_{\mathrm{n}}=$ $142,600-92,000)$ along with unimer peak. This result suggests that the polymerization of methacryloyl group in the core of the acetal-PEG- $b$-PLA-MA micelle occurred effectively by irradiation of plasma jets. This result is consistent with the conversion of 
methacryloyl group in the core of the acetal-PEG- $b$-PLA-MA micelle (Figure 4).

\subsection{Characterization of core-polymerzied micelles}

The size distribution of the core-polymerized acetal-PEG- $b$-PLA-MA micelle prepared using plasma irradiation for $40 \mathrm{~min}$ is shown in Figure 6 . The core-polymerized acetal-PEG- $b$-PLA-MA micelle as control sample was also prepared by thermal-induced free radical polymerization using AIBN for $24 \mathrm{~h}$ at $60{ }^{\circ} \mathrm{C}$ (ca. $100 \%$ conversion). As seen in Figure 6, increase in the average diameter and PDI were observed for control core-polymerized acetal-PEG- $b$-PLA-MA micelle $(62.3 \mathrm{~nm}, 0.236)$ compared with those of micelle before polymerization $(43.9 \mathrm{~nm}, 0.144$ in Figure 3 ). This is presumably due to the loading of AIBN into the core using organic solvent and/or the heating at $60{ }^{\circ} \mathrm{C}$ during polymerization process for $24 \mathrm{~h}$. In sharp contrast, no remarkable change in the average size and PDI $(51.4 \mathrm{~nm}$, 0.083 ) was observed for the core-polymerized acetal-PEG- $b$-PLA-MA micelle prepared using plasma irradiation.

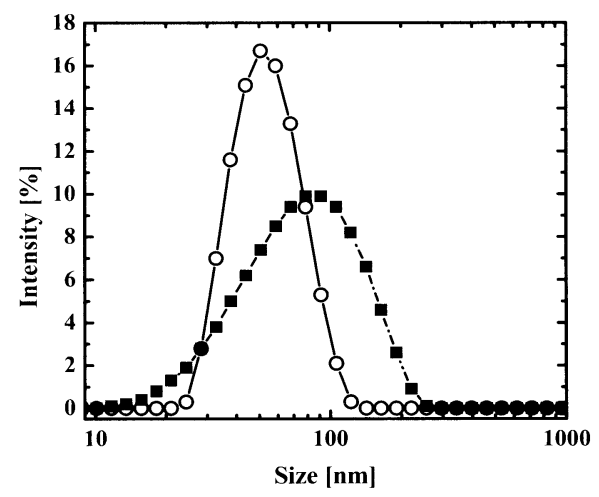

Figure 6. Size distribution of core-polymerized acetal-PEG- $b$-PLA-MA micelles prepared by irradiation of plasma for $40 \mathrm{~min}(\circ)$ and thermal-induced free radical polymerization $(\mathbf{a})$.

To clarify the effect of the core-polymerization on the stability of the micelles, the measurements of the light scattering intensity of the core-polymerized acetal-PEG- $b$-PLA-MA micelles were carried out in aqueous-THF solution $(33 \% \mathrm{v} / \mathrm{v})$. Figure 7 shows the change in light scattering intensity of micelle solution versus irradiation time $(0,20$, and $40 \mathrm{~min}$ ) of plasma jets. The each light scattering intensity of micelle solutions was normalized by the light scattering intensity of micelle solution in pure water $(0.5 \mathrm{mg} / \mathrm{mL})$, as defined 1 . The relative light scattering intensity of acetal-PEG- $b$-PLA micelles was less than 0.3 in aqueous-THF solution even after $40 \mathrm{~min}$. This fact suggests that the stabilization of acetal-PEG- $b$-PLA micelle could not be attained by simple irradiation of plasma jet, due to the lack of plasma-sensitive methacryloyl group, leading to the dissociation of the acetal-PEG- $b$-PLA micelle. In the case of core-polymerized acetal-PEG- $b$-PLA-MA micelles, the relative light scattering intensity of the micelle solution was dependent on plasma irradiation time, thus, significant high relative light scattering intensity $(0.55)$ was still observed after irradiation for $40 \mathrm{~min}$. This result indicates that acetal-PEG- $b$-PLA-MA micelle irradiated with plasma jets maintained the micellar structure even in aqueous-THF solution. Additionally, the CAC value of core-polymerized acetal-PEG- $b$-PLA-MA micelle prepared by the irradiation of plasma jets for 40 min was 1.5 $\mathrm{mg} / \mathrm{L}$ as determined by pyrene fluorescence probe method, and it was lower than that of acetal-PEG- $b$-PLA-MA micelle prepared without plasma irradiation $(2.4 \mathrm{mg} / \mathrm{L})$ (data not shown). On the basis of these results, acetal-PEG- $b$-PLA-MA micelle prepared by irradiation of plasma jet for 40 min showed the high stability against aqueous-THF solution and low CAC value.

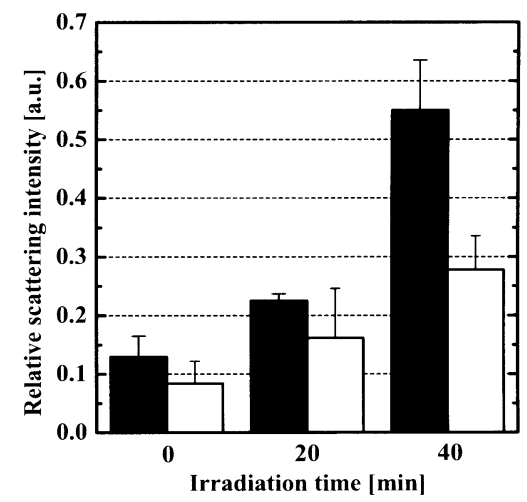

Figure 7. The change in light scattering intensity of acetal-PEG- $b$-PLA-MA (black) or acetal-PEG- $b$-PLA (white) micelle in aqueous-THF solution versus plasma irradiation time ( $n=3 \pm S D$.).

\section{Conclusions}

In conclusion, we describe the preparation and characterization of the core-polymerized acetal-PEG- $b$-PLA-MA micelle using the irradiation of plasma jets. Based on these systematic evaluations, core-polymerized acetal-PEG- $b$-PLA-MA micelle revealed the high 
stability against aqueous-THF solution and low CAC value without change in the size. This is the first demonstration of stabilizing biodegradable polymeric micelles by atmospheric plasma jets in the absence of any radical initiators. Additionally, this process is extremely simple and mild conditions compared with the chemically process. Therefore, this approach is promising candidate for the creation of the drug delivery carriers in vivo.

\section{References}

1. K.J. Zhu, S. Bihai, Y. Shilin, J. Polym. Sci.: Part A: Polym. Chem., 27, (1989) 2151-2159.

2. S.A. Hagan, A.G.A. Coombes, M.C. Garnett, S.E. Dunn, M.C. Davies, S.E. Harding, S. Purkiss, P.R. Gellert, Langmuir, 12, (1996), 2153-2161.

3. Y. Yamamoto, Y. Nagasaki, Y. Kato, Y. Sugiyama, K. Kataoka, J. Control. Rel., 77, (2001) 27-38.
4. Y. Nagasaki, T. Okada, C. Scholz, M. Iijima, M. Kato, K. Kataoka, Macromolecules, 31, (1998) 1473-1479.

5. A. Guo, G. Liu, J. Tao, Macromolecules, 29, (1996), 2487-2493.

6. A. Rösler, G.W.M. Vandermeulen, H.-A. Klok, Adv. Drug Deliv. Rev., 53, (2001) 95-108.

7. K. Kitano and S. Hamaguchi, Oyobutsuri, 77 (4), (2008) 383-389 (in Japanese).

8. H. Furusho, D. Miyamoto, K. Kitano, S. Hamaguchi, Y. Nagasaki, J. Photopoly. Sci. Tech., 20 (2), (2007) 229-233.

9. M. Iijima, Y. Nagasaki, T. Okada, M. Kato, K. Kataoka, Macromolecules, 32, (1999) 1140-1146.

10. J.-H. Kim, K. Emoto, M. Iijima, Y. Nagasaki, T. Aoyagi, T. Okano, Y. Sakurai, K. Kataoka, Polym. Adv. Tec., 10, (1999) 647-654.

11. M. Wilhelm, C.L. Zhao, Y. Wang, R. Xu, M.A. Winnik, Macromolecules, 24, (1991) 1033-1040. 